\title{
Perceived vibration and the loudness of low-frequency tones
}

\author{
MIGUELINA GUIRAO and JOSÉ ANTONIO VALCIUKAS \\ Laboratory of Psychophysics, Harvard University, Cambridge, Massachusetts 02138
}

\begin{abstract}
Vibration and low-frequency tones were scaled for loudness by two numerical estimation procedures and by cross-modality matching. The same ranges of frequencies, from 30 to $250 \mathrm{~Hz}$, were delivered to the ear and to the fingertip. For vibratory loudness, two sets of power functions were obtained, of which the low-frequency set was somewhat steeper. Tonal loudness gave a family of power functions of approximately the same slope at all the frequencies tested. For frequencies above $100 \mathrm{~Hz}$, the growth of loudness is about the same for both modalities. Below this frequency, vibratory loudness grows more rapidly than tonal loudness.
\end{abstract}

The subjective characteristics of vibratory "loudness," "pitch," size, localization, and other attributes have been compared with auditory attributes principally by Geldard (1940) and Katz (1920). Békésy $(1958,1959,1960)$ noted that some dynamic properties of sinusoidal waves, such as traveling waves, serve as stimuli for both auditory and tactile senses. He also found common features in the mechanical properties of the receptor organs, the neural structures, and the subjective characteristics of the two sensory systems. For a very sensitive part of the skin like the fingertip, Békésy showed that the grow th of the sensory intensity of a vibratory stimulus is much like the growth of loudness in hearing. Stevens (1959b), comparing the sensory magnitude of vibratory and auditory stimuli, concluded that the ear behaves somewhat like a compressor for loudness, and that the skin of the fingertip responds rather linearly to physical vibration. $\mathrm{He}$, too, suggested that the two systems may be mediated by similar processes.

In an effort to understand how the ear and the skin gauge an increase in the magnitude of the physical intensity of the stimulus, we have compared the loudness of low-frequency tones with the magnitude of the sensation produced by sinusoidal vibration delivered to the fingertip. More specifically, the present study compares the growth of perceived vibration and loudness for the same frequencies, between 15 and $250 \mathrm{~Hz}$, applying the same methods-magnitude estimation, magnitude production, and cross-modality matching-and using the same subjects.

\section{METHODS}

\section{Subjects}

Students and staff members served as experimental subjects.

The present address of the authors: Laboratorio de Investigaciones Sensoriales, CONICET and Facultad de Medicina, Universidad de Buenos Aires. Buenos Aires, Suc. 53, C.C. 53, Argentina. This work was supported by NIH Grant NS-02974, Laboratory of Psychophysics Report No. PPR-337.
None had had previous experience with psychophysical judgments of vibration. Ten subjects participated in the series of magnitude estimations of perceived vibration and loudness. Five of the 10 also participated in the series of magnitude productions and cross-modality matchings.

\section{Apparatus}

All the experiments were performed with the subjects in a soundproofed booth. A General Radio oscillator, Model 1310, produced the vibrations and the auditory signals. An electronic switch (Grason-Stadler Model 829-S-14) interrupted the signal in a sequence of $2 \mathrm{sec}$ on and $1 \mathrm{sec}$ off. The intensity levels of both vibratory and auditory signals were attenuated with two Hewlett-Packard attenuators: a Model $350 \mathrm{D}$ for vibration and a Model $350 \mathrm{~A}$ for tone. The signals were amplified by an Altex Model A 341 for vibration and a Grason-Stadler 1293 for tone. The amplitied tone was tiltered by an Allison variable filter. In the experiments with cross-modality matching, the subjects adjusted the amplitude of the tone by means of a sone potentiometer. A Gnodman vibrator. Model 390, was used to generate mechanical vibrations. A lightweight vibrator was used in an effort to avoid the propagation of mechanical vibrations beyond the limits of the stimulation area. This was the same apparatus with the same contactor area used by S. S. Stevens in previous studies (Stevens, 1959b). To minimize any possible leakage, the base and body of the apparatus were covered by several layers of padding. The intensities extended over about $45 \mathrm{~dB}$. The vibration generator was silent at all intensity levels at the lower frequencies, but at high-intensity levels of the higher frequencies, some hum was unavoid able. To attenuate that auditory cue, the subjects wore both antinoise ear plugs and an unwired headphone.

The active part of the vibrator was a metal rod, $3 \mathrm{~mm}$ in diam, with a round, dull tip approximately $0.02 \mathrm{~cm}^{2}$ in area. The amplitude of the vibration was calibrated by a method similar to that used by Geldard (1940). The tip of the vibrator was observed through a dissecting microscope while in operation, and its excursion was then measured in microns against a calibrated grid. During the calibration, the tip of the vibrator was not loaded with the skin.

The low-frequency tones were delivered to a specially calibrated earphone (Grason-Stadler, Model TDH 49, 10 ohms), whose calibration curve was flat from 10 to $1,000 \mathrm{~Hz}$. Stimulation was binaural.

The subject sat in a comfortable chair and rested his arm and hand on a cushioned board in which there was an opening containing a ring, $15 \mathrm{~mm}$ in diam. The subject touched lightly, with the fingertip without pressing, the tip of the vibrator located at the center of the ring.

For the vibratory stimuli, the smallest intensity level used ranged from a few microns above threshold at $30 \mathrm{~Hz}$ to about 40 microns 


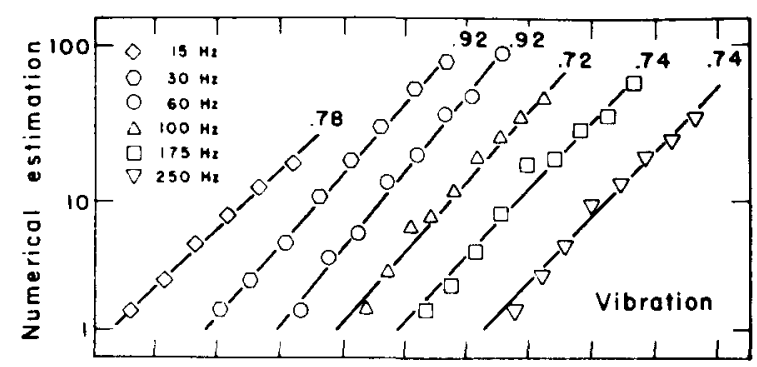

Peak-to-peak amplitude of vibration in microns (relotive scale)

Figure 1. Magnitude estimations of perceived vibration. The points are the geometric means of 20 estimates given by 10 subjects. All functions are shifted to an approximately equal initial point. The exponents are given at the top.

above threshold at $175 \mathrm{~Hz}$. For loud ness, the softest tone was $56 \mathrm{~dB}$ SPL at $30 \mathrm{~Hz}$.

\section{Procedures}

For magnitude estimation, the subject was instructed in writing to estimate either the intensity of the vibration or the loudness of a tone by assigning a number that seemed to him to match his subjective impression. The subjects chose their own modulus, and no suggestion was made that the numbers assigned should be proportional to the standard stimulus. When magnitude production was used, the instruction was the same except that the subject was instructed to adjust the intensity of the vibration or the tone to match a number given by the experimenter. When the task was to match loudness to vibration, the subject was presented with tones at several intensity levels and he was instructed to adjust the vibration to match the apparent intensity of each tone.

For both modalities, the frequencies selected were $30,60,100$. 175 . and $250 \mathrm{~Hz}$, plus $15 \mathrm{~Hz}$ which was used with vibration only. All the selected frequencies were used in the experiments with magnitude estimation. With magnitude production, only 30,100 , and $250 \mathrm{~Hz}$ were used. Simultaneous cross-modality matching was performed with two stimulus frequencies, 100 and $250 \mathrm{~Hz}$. The subjects controlled the intensity of the sound by turning the knob of the sone potentiometer so as to adjust the intensity of the tone to the intensity of the vibrator. Successive cross-modality matching was performed with a $100 \cdot \mathrm{Hz}$ signal. Conditions were the same as described before, except that the 2-sec signals were presented in succession with an interval of 1 sec.

\section{The Magnitude of Perceived Vibration}

In Figures 1 and 2 are plotted the geometric means of the estimates of perceived vibration as a function of the peak-to-peak amplitude of the sinusoidal vibration. In agreement with results reported by Stevens $(1959 \mathrm{a}, \mathrm{b})$, power functions related the subjective magnitude of vibration to the amplitude of vibration. As Figure 1 shows, 30 and $60 \mathrm{~Hz}$ gave the same exponent, about 0.9 , whereas the three higher frequencies gave a lower exponent, about 0.7. Similar results were obtained by Stevens (1968), who predicted that the exponents for vibration would become lower as the frequency of the stimulus was increased. An exponent of 0.78 was obtained at $15 \mathrm{~Hz}$, where the sensation of vibration may no longer be present.
Figure 2 shows arithmetic means for magnitude production of perceived vibration. The exponents are on the average higher, by 0.6 , than those obtained by magnitude estimation. This difference in the value of the exponents is expected with magnitude production (Stevens, 1971). For the $100-\mathrm{Hz}$ tone, the exponent changed drastically with the method used. Because the exponent of 1.6 obtained with magnitude production seemed to us very large relative to the $\mathbf{0 . 7 2}$ obtained with magnitude estimation, we ran another experiment with different subjects and obtained the same results. With both procedures, the exponent for vibration was higher for $30 \mathrm{~Hz}$ than for $250 \mathrm{~Hz}$. However, at the three frequencies tested, the average of the exponents obtained by magnitude estimation and production decreased with the frequency, though not very dramatically. Moreover, the average value-a little above unity-agrees well with the exponents for the subjective vibration functions presented by Verrillo (1969) for approximately the same frequencies. This author stimulated the thenar eminence and used both magnitude estimation and magnitude production procedures. He reported an average exponent of about 1.0 up to $350 \mathrm{~Hz}$. It should be mentioned that these results compare closely with the exponent of 1.1 obtained by J. C. Stevens and Mack (1959) when they stimulated the palm of the hand with static pressure. In a previous study, Valciukas and Guirao (1972), using gas pressure to stimulate the palm, generated power functions with exponents of about 1.0. Thus, subjective response of the skin to increasing intensity of pressure may turn out to give similar exponents, regardless of whether the pressure is static or dynamic.

\section{The Quality of Perceived Vibration}

Several experimenters (Békésy, 1960; Geldard, 1940) have suggested that the quality of the vibratory

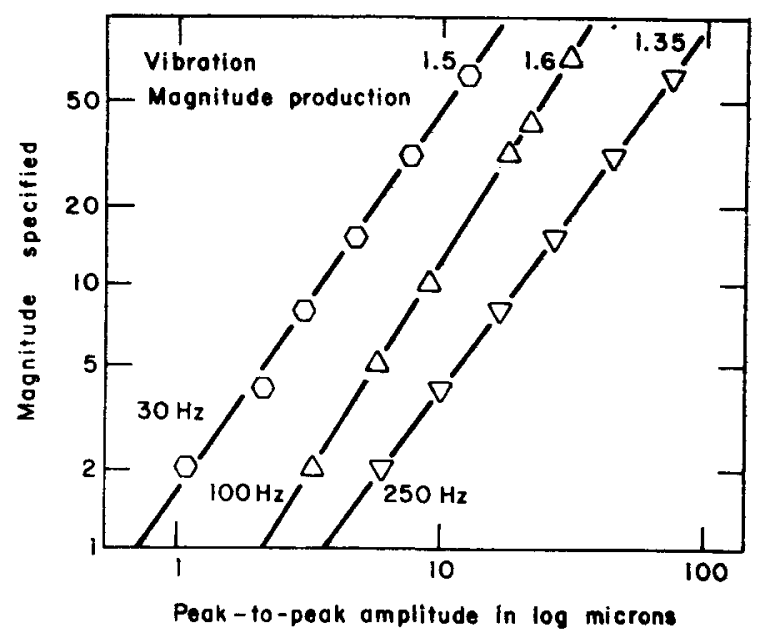

Figure 2. Magnitude production of percelved vibration. The points are the arithmetic means of $\mathbf{1 0}$ adjustments by five subjects. 
stimulus may vary with the frequency of the stimulus. Our subjects observed that at the lowest frequency, $15 \mathrm{~Hz}$, the tip of the vibrator seemed to be large and dull. whereas at higher frequencies it felt small and sharp. In addition, the lower frequencies were experienced more as a repetitive or intermittent stimulus, rather than as a vibratory stimulus. Geldard (1940), Katz (1920), and Mach and Busler (quoted by Katz, 1920) have suggested that at very low frequencies the sensation of vibration may no longer be present. During the experimental sessions, we asked the subjects to say when the stimulus began to be felt as a vibration. The median stimulus value at which they began to perceive vibration was $32 \mathrm{~Hz}$. Verrillo (1962) measured thresholds for vibration on the fleshy part of the hand and concluded that below $40 \mathrm{~Hz}$, the threshold for vibration was independent of stimulus frequency and contactor area. It is tempting, therefore, to speculate that the differences in the slopes of Figure 1 do indeed reflect these qualitative changes, i.e.. that the increase in exponent between 15 and $30 \mathrm{~Hz}$ may reflect the repetition rate quality, and that only above about $60 \mathrm{~Hz}$ is the stimulus perceived as a vibration.

\section{Loudness}

Figures 3 and 4 show the means of loudness estimates as a function of the sound pressure level of the pure tone. The five power functions in Figure 3, obtained by magnitude estimation, all have approximately the same exponent, 0.6. As with perceived vibration, the exponents obtained with magnitude production were higher, by about 0.5 , than those obtained with magnitude estimation. No important change in exponent was observed as a function of frequency with either of the two procedures.

Stevens $(1966,1968)$ collated data from studies by Churcher and King (1937). Fletcher and Munson (1933). Kingsbury (1927), and Robinson and Dadson (1956) to calculate the slopes of the loudness functions for low-frequency tones. He drew four sets of

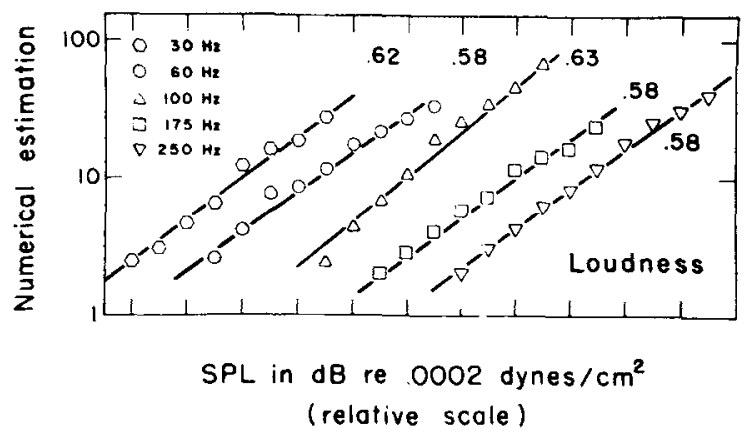

Figure 3. Magnitude estimations of the loudness of pure tones. The points are the geometric means of 20 estimates given by 10 subjects.

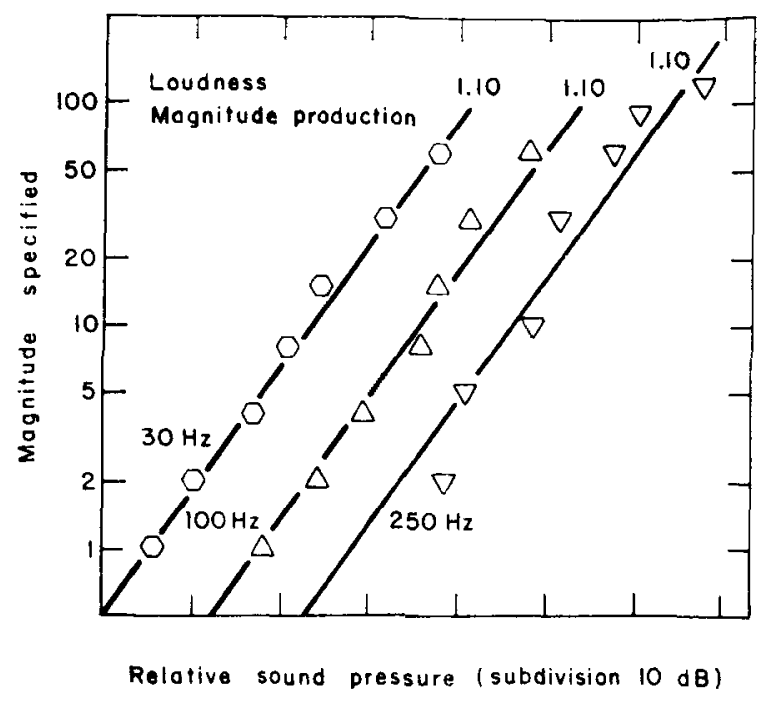

Figure 4. Magnitude productions of loudness. The points are the arithmetic means of $\mathbf{1 0}$ adjustments by five subjects.

functions, ranging from 33.3 to $350 \mathrm{~Hz}$, with exponents that decreased with increasing frequencies. His calculations were based on data obtained from matching and tracking procedures. Schneider, Wright, Edelheit, Hock, and Humphrey (1971) collected data under magnitude estimation for tones between 100 and $2,500 \mathrm{~Hz}$ and seemed to arrive at the same conclusion for tones below $500 \mathrm{~Hz}$. However, a look at the group data of their Figure 1 gives the distinctive impression that the functions up to $300 \mathrm{~Hz}$ are parallel and of the order of 0.6. Also, the upper portion of the loudness function reported by Hellman and Zwislocki (1968) gives similar slopes for both 100 and $250 \mathrm{~Hz}$. Our Figure 3 indicates that the exponent of 0.6 . proposed by Stevens for a pure tone at $1.000 \mathrm{~Hz}$, holds well into the range of low frequencies.

\section{Cross-Modality Matching}

The task of trying to verify the relation between auditory and vibratory loudness by cross-modality matching posed several problems. During pilot experiments, the subjects reported that the localization and quality of the sensations for both stimuli seemed to vary drastically during simultaneous matching. When the intensity of either of the stimuli was varied, the vibration was sometimes localized at the finger and the tone at the ear, or a new sensation of tone-vibration might fill the entire space between the ear and the arm. This tone-vibration sensation seemed to travel along the arm as the intensity of the tone (or vibration) was varied with the potentiometer. These localization changes made the subjects task extremely difticult. especially when they attempted to adjust vibration to match loudness. When the task called for adjusting the loudness to match the vibration. although some of these effects 


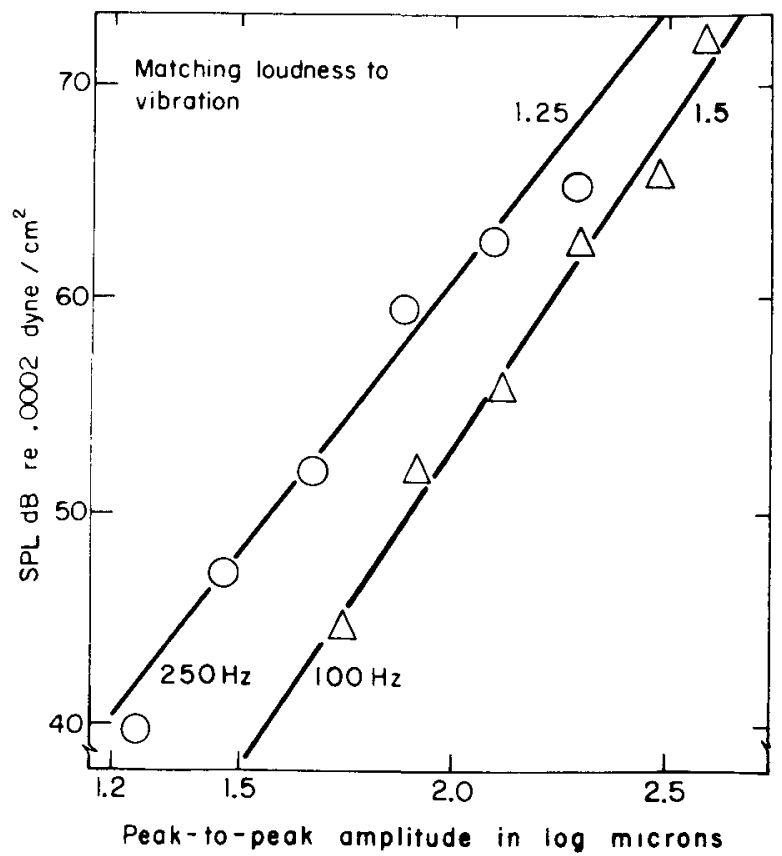

Figure 5. Matching loudness to vibration. The points are the arithmetic means of 10 loudness adjustments by five subjects.

crept in at the highest levels of both signals, the changes were not so marked as to confuse the subjects.

In addition to these problems, matching vibration to loudness presents other difficulties. For example, Reynolds and Stevens (1960) noted that when vibration is matched to loudness, the range that is available for vibration is considerably shorter than the range of available loudness. All considered in the first place, we did try simultaneous matching of loudness to vibration. With loudness under the subjects' control, the exponent should be predictable from the ratio of the exponents for vibration and loudness obtained by numerical estimation. For $100 \mathrm{~Hz}$, ratios

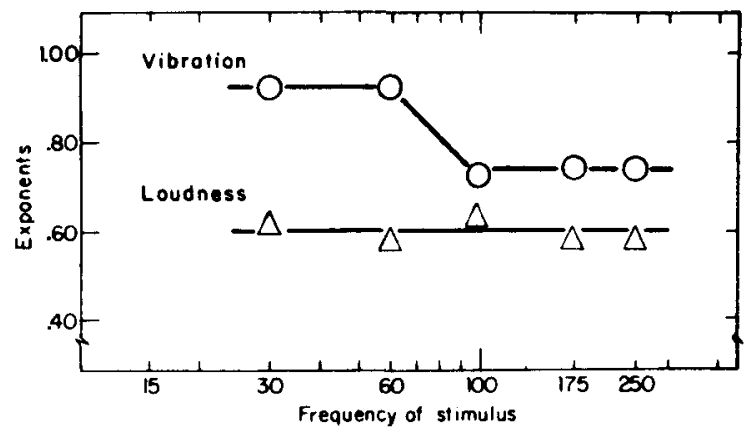

Figure 6. Comparison of the slopes (exponents) for perceived vibration and loudness. Above $60 \mathrm{~Hz}$, the exponents of the two functions are similar in size. Below that frequency, vibration functions are steeper, indicating that vibratory loudness increases more rapidly than tonal loudness. are about 1.1 and 1.5 by magnitude estimation and magnitude production, respectively; for $250 \mathrm{~Hz}$, it is about 1.25 by both methods. As can be observed in Figure 5 , the predicted ratio for $250 \mathrm{~Hz}$ was verified experimentally. The exponent for $100 \mathrm{~Hz}$ corresponds to the ratio predicted by the production method. Finally, we ran another experiment with a $100-\mathrm{Hz}$ signal to check whether the adjustments would be easier with successive than with simultaneous matching. The task seemed to be less confusing as reported by the subjects. Under such conditions, the exponent 1.1 was obtained which is the ratio predicted by magnitude estimation.

\section{Comparison Between Vibratory and Auditory Loudness}

The loudness and vibration functions obtained by numerical estimation for the five frequencies delivered to the ear and to the fingertip are compared in Figure 6. The vibration functions are consistently steeper; their exponents average 0.3 higher than those of tonal loudness at the two lower frequencies, 30 and $60 \mathrm{~Hz}$. Up to $60 \mathrm{~Hz}$, these results support Békésy's observations that the sensation grows more rapidly on the skin than in the ear. For the other three frequencies, 100,175 , and $250 \mathrm{~Hz}$, the difference was negligible, about 0.1 . The comparison described here supports Stevens' (1959a) observation that the skin behaves like a linear system up to about $100 \mathrm{~Hz}$, and that above that frequency, the skin, like the ear, seems to behave as a compressor.

\section{REFERENCES}

BÉkÉsy, G. von. Funneling in the nervous system. Journal of the Acoustical Society of America, 1958, 30, 399-412.

BÉKÉSY, G. von. Similarities between hearing and skin sensations. Psychological Review, 1959, 66, 1-22.

BÉKÉsY, G. voN. Experiments in hearing. New York: McGraw.Hill, 1960.

Churcher, B. G., \& King, A. J. The performance of noise meters in terms of the primary standard. Journal of the Institute of Electrical Engineers (London), 1937, 81, 57-90.

Fletcher, H., \& Munson, W. A. Loudness, its definition, measuring, and calculation. Journal of the Acoustical Society of America, 1933, 5. 82-108.

GELDARD, F. A. The perception of mechanical vibration. Journal of General Psychology, 1940, 22, 243-308.

Hellman, R. P., \& Zwislocki, J. J. Loudness determination at low sound frequency. Journal of the Acoustical Society of America, 1968, 43, 60-64.

KATZ, D. Die Erscheinungsweisen der Tasteindrücke. Rostock: Kommissionsverlag von $\mathbf{H}$. Warkentien, 1920.

Kingsbury, B. A. A direct comparison of the loudness of pure tones. Physics Review, 1927, 29, 588-600.

Reynolds, G. S., \& Stevens. S. S. Binaural summation of loudness. Journal of the A coustical Society of America, 1960. 32. 1337.1344 .

Robinson, D. W., \& Dadson, R. S. A re-determination of the equal-loudness relations for pure tones. British Journal of Applied Physics, 1956, 7, 166-181.

Schneider, B., Wright, A. A., Edelheit, W., Hock, P., \& 
Humphrey, C. Equal loudness contours derived from sensory magnitude judgments. Journal of the Acoustical Society of America, 1971, 51. 1951-1959.

Stevens, J. C.. \& MACK. J. D. Scales of apparent force. Journal of Experimental Psychology, 1959, 58, 405-413.

STEVENS. S. S. Cross-modality validation of subjective scales for loudness. vibration and electric shock. Joumal of Experimental Psychology, 1959, 57, 201-209. (a)

Stevens. S. S. Tactile vibration: Dynamics of sensory intensity. Journal of Experimental Psychology, 1959, 57, 210-218. (b)

Stevens. S. S. Power-group transformations under glare. masking. and recruitment. Journal of the Acoustical Society of America. 1966. 39. 725-735.

Stevens. S. S. Tactile vibration: Change of exponent with frequency. Perception \& Psychophysics, 1968, 3, 223-228.
Stevens, S. S. Issues in psychophysical measurement. Psichological Review', 1971, 78, 426-450.

Valciukas. J. A., \& Guirao, M. Inhibition of response to gas pressure on the skin. Proceedings of the $X X$ th International Congress of Psychology. Tokyo, August 13-19, 1972.

VERRILLO, R. T. Investigation of some parameters of the cutaneous threshold for vibration. Journal of the Acoustical Society of America, 1962, 34, 1768-1773.

Verrillo, R. T. Sensation magnitude of vibratory stimuli. Perception \& Psychophysics, 1969, 6, 366-373.

(Received for publication December 24, 1973; revision received January 10,1975 .) 